\title{
Two-way relaying using constant envelope modulation and phase-superposition-phase-forward
}

\author{
Huai Tan and Paul Ho*
}

\begin{abstract}
In this article, we propose the idea of phase-superposition-phase-forward (PSPF) relaying for 2-way 3-phase cooperative network involving constant envelope modulation with discriminator detection in a time-selective Rayleigh fading environment. A semi-analytical expression for the bit-error-rate (BER) of this system is derived and the results are verified by simulation. It was found that, compared to one-way relaying, 2-way relaying with PSPF suffers only a moderate loss in energy efficiency (of $1.5 \mathrm{~dB}$ ). On the other hand, PSPF improves the transmission efficiency by $33 \%$. Furthermore, we believe that the loss in transmission efficiency can be reduced if power is allocated to the different nodes in this cooperative network in an 'optimal' fashion. To further put the performance of the proposed PSPF scheme into perspective, we compare it against a phase-combining phase-forward technique that is based on decode-and-forward (DF) and multi-level CPFSK re-modulation at the relay. It was found that DF has a higher BER than PSPF and requires additional processing at the relay. It can thus be concluded that the proposed PSPF technique is indeed the preferred way to maintain constant envelope signaling throughout the signaling chain in a 2-way 3 phase relaying system.
\end{abstract}

Keywords: 2-way relaying, phase-only-forward, decode-and-forward, cooperative communications, constant envelope modulation, continuous phase modulation, CPFSK, discriminator detection

\section{Introduction}

Cooperative transmission is a cost effective way to combat fading because it creates a virtual multiple-input-multipleoutput (MIMO) communication channel without resorting to mounting antenna arrays at individual nodes [1,2]. Earlier researches on cooperative transmission focus on one-way relaying with amplify-and-forward (AF) and decode-and-forward (DF) protocols [3-5]. Orthogonal time-slots are employed by the source and the relay to allow the destination node to obtain independent faded copies of the same message for combining purpose $[3,4]$. The creation of these orthogonal time slots reduces the throughput of the system [6]. For example, the so-called Protocol II in [7] has a throughput of $1 / N$ message/slot, where $N$ is the number of relays in the system.

To improve the transmission efficiency of cooperative communication, two-way relaying is proposed [8-12]. For example in [12], a two-way relay network where two users exchange information with the assistance of an

\footnotetext{
* Correspondence: paulho@cs.sfu.ca

School of Engineering Science, Simon Fraser University, Burnaby, British Columbia V5A 156, Canada
}

intermediate relay node was considered. Specifically, the authors consider the so-called decode-superposition-forward (DSF) and decode-XOR-forward (DXF) protocols for 2-way 3-phase relaying. These protocols can support bi-lateral transmission over three orthogonal time slots, leading to an improved throughput of $2 / 3$ message/slot, i.e., a $33 \%$ improvement over 1-way relaying with a single relay.

The signals transmitted by all three nodes in the system in [12] are QAM-type linear modulations. While linear modulation has many desirable features, it imposes a relatively stringent requirement on amplifier linearity. This is especially true in the case of DSF, where the transmitted signal constellation at the relay is essentially the superposition of two constituent QAM constellations. In contrast, constant envelope modulation enables the use of inexpensive nonlinear (Class C) power amplifiers. These modulations are widely used in public safety (police, ambulance) and private mobile communication systems (taxi, dispatch, courier fleets), even though they are, in general, not as bandwidth efficient as QAM modulations. The use of constant envelope modulations in 
cooperative communications had been considered in [13-15]. Specifically, in [15], continuous-phase frequencyshift-keying (CPFSK) and phase-forward was proposed for 2-node MRC-type cooperative communication system with time-selective Rayleigh fading and discriminator detection. The authors reported that PF has a lower BEP than decode-and-forward. It also delivers the same performance as amplify-and-forward when dual-antenna selection is available at the relay. They concluded that PF is a cost-effective alternative to AF and DF, since it does not need signal regeneration at the relay nor does it need expensive linear amplifiers.

In this article, we consider the application of CPFSK and phase-forward in a 2-way 3-phase cooperative communication system with time-selective Rayleigh fading. A major contribution is in the development of a phasesuperposition phase-forward (PSPF) strategy that maintains the constant envelope property at the relay without resorting to any intermediate decoding. The usefulness of the proposed PSPF scheme is confirmed via a semi-analytical bit-error-rate (BER) analysis, as well as comparing it against one-way relaying and against a 2-way 3-phase strategy based on decode-and-forward and multi-level CPFSK re-modulation at the relay.

The article is organized as follows. We first describe in Section 2, the signal and system model for the proposed PSPF relaying scheme and competing Decode and Forward (DF) schemes based on multilevel CPFSK re-modulation at the relay. The detection and combining strategies are presented in Section 3, followed by a discussion of implementation issues in Section 4. The BER of the proposed scheme is analyzed in Section 5, and the companion numerical results provided in Section 6. Finally, conclusions of this investigation are given in Section 7.

We adopt the following notations/definitions throughout the article: $j^{2}=-1 ;(\cdot)^{* *}$ and $|\cdot|$ denote, respectively, the conjugate and magnitude of a complex number; $(\cdot)^{\mathrm{T}}$ and $(.)^{\dagger}$ represent, respectively, the regular and Hermitian transposes of a matrix; $E[\cdot]$ is the expectation operator; $\frac{1}{2} E\left[|x|^{2}\right]$ the variance of a zero-mean complex random variable $x$ with independent and identically distributed (i.i.d.) real and imaginary components; $C N\left(\mu, \sigma^{2}\right)$ refers to a complex Gaussian random variable with mean $\mu$ and variance $\sigma^{2} ; \operatorname{sinc}(x)=\sin (\pi x) /(\pi x)$; and $\dot{x}$ the derivative of $x$.

\section{3-phase 2-way cooperative communication system model}

We consider a 3-phase 2-way relay cooperative communication system consisting of three nodes: user $A$ and its bilateral partner user $B$, as well as a relay $R$. All nodes operate in half duplex mode. The system diagram is shown in Figure 1. During the first phase, $A$ transmits

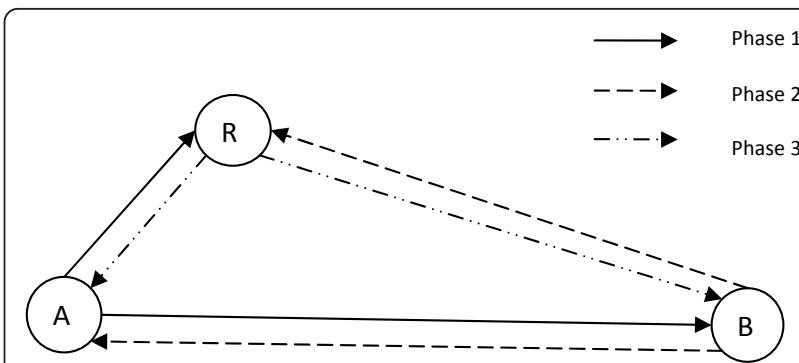

Figure 1 3-phase 2-way relaying system model.

its data to $B$, while $B$ and $R$ listen. The received signals at $R$ and $B$ are

$$
y_{R, 1}(t)=g_{A R}(t) x_{A}(t)+n_{R, 1}(t)
$$

and

$$
y_{B, 1}(t)=g_{A B}(t) x_{A}(t)+n_{B, 1}(t)
$$

where $x_{A}(\mathrm{t})$ is the signal transmitted by $A, n_{R, 1}(t)$ and $n_{B, 1}(t)$ the zero-mean complex additive white Gaussian noise (AWGN) terms at $R$ and $B$ in the first phase, and $g_{A R}(t)$ and $g_{A B}(t)$ the zero-mean complex Gaussian processes that represent Rayleigh fading in the $A-R$ and $A-B$ links.

In the second phase, it is $B$ 's turn to transmit its data to $A$. This time, both $A$ and $R$ are in the listening mode. The received signals at $R$ and $A$ are

$$
y_{R, 2}(t)=g_{B R}(t) x_{B}(t)+n_{R, 2}(t)
$$

and

$$
\gamma_{A, 2}(t)=g_{B A}(t) x_{B}(t)+n_{A, 2}(t)
$$

where $x_{B}(t)$ is the signal transmitted by $B, n_{R, 2}(t)$ and $n_{A, 2}(t)$ the AWGNs at $R$ and $A$ in the second phase, and $g_{B R}(t)$ and $g_{B A}(t)$ the zero-mean complex Gaussian processes that represent Rayleigh fading in the $B-R$ and $B-A$ links.

Finally in the last phase, only $R$ transmits, both $A$ and $B$ listen. The received signals at $A$ and $B$ are

$$
\gamma_{A, 3}(t)=g_{R A}(t) x_{R}(t)+n_{A, 3}(t)
$$

and

$$
y_{B, 3}(t)=g_{R B}(t) x_{R}(t)+n_{B, 3}(t)
$$

where $x_{R}(t)$ is the signal transmitted by $R, n_{A, 3}(t)$ and $n_{B, 3}(t)$ the complex AWGNs at $A$ and $B$ in the third phase, and $g_{R A}(t)$ and $g_{R B}(t)$ the zero-mean complex Gaussian processes that represent Rayleigh fading in the $R-A$ and $R-B$ links. In this investigation, we assume the six fading processes in (1) to (6) are statistically 
independent. In addition, all the six noise terms in (1) to (6) are i.i.d.

In this article, all the transmitted signals $x_{A}(t), x_{B}(t)$, and $x_{R}(t)$ are constant envelope signals.

Specifically, the former two are CPFSK signals of the form

$$
x_{i}(t)=e^{j \theta_{i}(t)} ; \quad i \in\{A, B\},
$$

where

$$
\theta_{i}(t)=\pi h\left(\sum_{n=0}^{k-1} d_{i, n}\right)+\pi h d_{i, k} \cdot(t-k T) / T, \quad k T<t \leq(k+1) T,
$$

is the information carrying phase, with $d_{i, k} \in\{ \pm 1\}$ being the data bit in the $k$-th symbol interval for User $i$, $i \in\{A, B\}, h$ being the modulation index, and $T$ the bitduration. Note that the derivative of the information carrying phase is

$$
\dot{\theta}_{i}(t)=\pi h d_{i, k} / T, \quad k T<t \leq(k+1) T,
$$

which is proportional to the data bit $d_{i, k}$. This property is crucial in understanding the decision rule made by the discriminator detector presented in the next section. Another property of CPFSK that is important to the understanding of the results is the bandwidth of the signal. It is well known [16] that CPFSK signals, are in general, not band-limited. As such, a common practice is to adopt the frequency range that contains $99 \%$ of the total signal power as the bandwidth of the signal. This is referred to as the 99\% bandwidth [16]. As an example, consider the so-called minimum shift keying (MSK) scheme, i.e., CPFSK with $h=1 / 2$. Using the results from [17], the $99 \%$ bandwidth of MSK is found to be $1.1818 / T$.

\subsection{Phase superposition phase forward}

The signal transmitted by the relay, $x_{R}(t)$, assumes the following form

$$
x_{R}(t)=\exp \left\{j\left(\arg \left[\gamma_{R, 1}(t)\right]+\arg \left[\gamma_{R, 2}(t)\right]\right)\right\},
$$

where $\arg \left[y_{R, 1}(\mathrm{t})\right]$ and $\arg \left[y_{R, 2}(\mathrm{t})\right]$ are the phases of the signals $y_{R, 1}(\mathrm{t})$ and $y_{R, 2}(\mathrm{t})$, respectively. Note that $x_{R}(t)$ is both constant envelope and continuous-phase, just like the data signals $x_{A}(t)$ and $x_{B}(t)$. We thus call the forwarding strategy in (10) a phase superposition-phase-forward (PSPF) scheme. Since this phase superposition is equivalent to a multiplication of (the hard-limited versions of) the signals $y_{R, 1}(t)$ and $y_{R, 2}(t)$ in the time domain, the corresponding frequency domain convolution will lead to a spectrum expansion if the relay is destined to transmit without any bandwidth limitation.

One nice feature of the proposed PSPF technique is that constant envelope signaling is maintained at the relay without requiring it to perform any demodulation and re- modulation. A natural question to ask is, how does PSPF compare to decode-and-forward strategies that employ constant envelope signaling at the relay? To be able to answer this question, we introduce next the 3-level decode-and phase-forward (3-DPF) scheme and the alternate 4-level decode-and-phase-forward (A4-DPF) scheme as possible alternatives to PSPF. For both schemes, the relay first make decisions on User $A$ 's and User $B$ 's data based on the its received signals $y_{R, 1}(t)$ and $y_{R, 2}(t)$. It then applies the decisions, $\hat{d}_{A, n}$ and $\hat{d}_{B, n}$, to (7) and (8) to regenerate User $A$ 's and User $B$ 's signals according to $\hat{x}_{A}(t)=\exp \left\{j \hat{\theta}_{A}(t)\right\}$ and $\hat{x}_{B}(t)=\exp \left\{j \hat{\theta}_{B}(t)\right\}$.

\subsection{3-level decode and phase forward (3-DPF)}

With this decode and forward strategy, the relay adds the decoded phases in $\hat{x}_{A}(t)$ and $\hat{x}_{B}(t)$ synchronously to form the relay signal

$$
x_{R}(t)=\exp \left\{j\left(\hat{\theta}_{A}(t)+\hat{\theta}_{B}(t)\right)\right\}=\hat{x}_{A}(t) \hat{x}_{B}(t) .
$$

This signal is both constant envelope and continuousphase, just like the data signals $x_{A}(t)$ and $x_{B}(t)$. Furthermore, because of synchronous mixing, we can view $x_{R}(t)$ as a 3-level CPFSK signal with modulation index $h$ and symbol values $+2,0,-2$ that occur with priori probabilities $\frac{1}{4}, \frac{1}{2}, \frac{1}{4}$. The three signal levels and the corresponding priori probabilities are due to the fact that the decoded bits $\hat{d}_{A, n}$ and $\hat{d}_{B, n}$ at the relay are $\{ \pm 1\}$ binary random variables. Another consequence of synchronous phase mixing is that the bandwidth of $x_{R}(t)$ is less than the sum bandwidth of $\hat{x}_{A}(t)$ and $\hat{x}_{B}(t)$, even though $x_{R}(t)=\hat{x}_{A}(t) \hat{x}_{B}(t)$. Using MSK as example again, the sum bandwidth is two times $1.1818 / T$ or $2.3636 / T$. The 99\% bandwidth of the corresponding $x_{R}(t)$, on the other hand, is only $1.832 / T$.

\subsection{Alternate 4-level decode and phase forward (A4-DPF)}

In general, we can construct a constant-envelope relay signal based on the superposition of the decoded phases as follows

$$
x_{R}(t)=\exp \left\{j\left(w_{A} \hat{\theta}_{A}(t)+w_{B} \hat{\theta}_{B}(t)\right)\right\}
$$

where $w_{A}$ and $w_{B}$ are weighting coefficients $[9,10,12]$. In the case where $w_{A}=2$ and $w_{B}=1, x_{R}(t)$ becomes a conventional 4-level CPFSK scheme with modulation $h$ and symbol values $+3,+1,-1,-3$ all occurring with equal probability. This signal will have a wider bandwidth than the 3-level relay signal in the previous section but it also has the potential to provide a better BEP performance (typical power-bandwidth tradeoff). One thing though, 
the unequal weightings on the two decoded phases will translate into an asymmetric error performance at $A$ and $B$. This problem can be alleviated by alternating the weighting rules between even and odd time slots as follows:

$$
\begin{array}{ll}
w_{A}=2, w_{B}=1 ; & \text { even time slot }, \\
w_{A}=1, w_{B}=2 ; & \text { odd time slot. }
\end{array}
$$

We call this strategy alternate 4-level decode and phase forward or A4-DPF.

\section{Discriminator detection of the relay signals}

As shown in (1) to (6), the transmitted signals at $A, B$, and $R$, will in general, experience time-selective fading. This makes the implementation of coherent detection rather complicated. As such we consider the much simpler discriminator detector. This non-coherent detector does not need channel state information when making data decisions and it thus spares the receiver from performing complicated channel tracking and sequence detection tasks. Without loss of generality, we demonstrate in the following sections how User $A$ detects the data intended for it from User $B$, i.e., the $d_{B,}$ 's, using a discriminator detector. The detection of User $A$ 's data at Node $B$ follows exactly the same procedure. It is further assumed that ideal lowpass filters (LPF) are used to limit the amount of noise admitted into the detector, with the bandwidth of each receive LPF set to the $99 \%$ bandwidth of its incoming signal. As such, the noise processes in (1) to (6) are all band-limited white Gaussian noises.

\subsection{Detection of PSPF signals}

To see how discriminator detector works in the proposed PSPF system, we first rewrite the two received signals at the relay as

$$
\gamma_{R, 1}(t)=g_{A R}(t) \mathrm{e}^{j \theta_{A}(t)}+n_{R, 1}(t)=a_{R, 1}(t) \mathrm{e}^{j \psi_{R, 1}(t)}
$$

and

$$
\gamma_{R, 2}(t)=g_{B R}(t) \mathrm{e}^{\mathrm{j} \theta_{B}(t)}+n_{R, 2}(t)=a_{R, 2}(t) \mathrm{e}^{\mathrm{j} \psi_{R, 2}(t)},
$$

where $a_{R, 1}(t), a_{R, 2}(t), \psi_{R, 1}(t)$, and $\psi_{R, 2}(t)$ are the amplitudes and phases of the two signals. As stated in (10), the relay broadcasts

$$
\begin{aligned}
& x_{R}(t)=\exp \left\{j \theta_{R}(t)\right\}, \\
& \theta_{R}(t)=\psi_{R, 1}(t)+\psi_{R, 2}(t),
\end{aligned}
$$

to $A$ and $B$ in the last phase. Substituting (16) into (5), the received signal at $A$ during the third phase can now be written as

$$
\gamma_{A, 3}(t)=g_{R A}(t) \mathrm{e}^{j \theta_{R}(t)}+n_{A, 3}(t)=a_{A, 3}(t) \mathrm{e}^{j \psi_{A, 3}(t)},
$$

where $a_{A, 3}(t)$ and $\psi_{A, 3}(t)$ are, respectively, the amplitude and phase.

In order to detect the signal from $B$, User $A$ first removes its own phase $\theta_{A}(t)$ from $\psi_{A, 3}(t)$. The resultant complex signal is

$$
\begin{aligned}
& Y_{A, 3}(t)=a_{A, 3}(t) \mathrm{e}^{j \Psi_{A, 3}(t)}, \\
& \Psi_{A, 3}(t)=\psi_{A, 3}(t)-\theta_{A}(t) .
\end{aligned}
$$

It then combines $Y_{A, 3}(t)$, non-coherently, with the signal

$$
\gamma_{A, 2}(t)=g_{B A}(t) \mathrm{e}^{j \theta_{B}(t)}+n_{A, 2}(t)=a_{A, 2}(t) \mathrm{e}^{j \psi_{A, 2}(t)}
$$

from (4), where $a_{A, 2}(t)$ and $\psi_{A, 2}(t)$ are, respectively, the received signal amplitude and phase.

Specifically at the decision making instant, which is taken to be the mid-symbol position in each bit interval, the non-coherent detector adds the phase derivatives $\dot{\psi}_{A, 2}$ and $\dot{\Psi}_{A, 3}$ according to the maximal ratio combining principle [15]

$$
D=D_{2}+D_{3}
$$

Where

$$
\begin{aligned}
& D_{2}=2 a_{A, 2}^{2} \dot{\psi}_{A, 2}=\left(\gamma_{A, 2}^{*} \dot{y}_{A, 2}^{*}\right)\left(\begin{array}{ll}
0 & -j \\
j & 0
\end{array}\right)\left(\begin{array}{l}
y_{A, 2} \\
\dot{y}_{A, 2}
\end{array}\right), \\
& D_{3}=2 a_{A, 3}^{2} \dot{\Psi}_{A, 3}=\left(Y_{A, 3}^{*} \dot{Y}_{A, 3}^{*}\right)\left(\begin{array}{ll}
0 & -j \\
j & 0
\end{array}\right)\left(\begin{array}{l}
Y_{A, 3} \\
\dot{Y}_{A, 3}
\end{array}\right),
\end{aligned}
$$

and then makes a decision on the data bit in question, $d_{B}$, according to

$$
\hat{d}_{B, k}=\operatorname{sgn}(D) .
$$

An intuitive understanding of the above decision rule can be gained by considering the ideal situation where there are no fading and noise in all the links. In this case, the received phase derivatives at the relay and at node $A$ during the first and second phases of transmission are $\dot{\psi}_{R, 1}(t)=\pi h d_{A, k} / T$ and $\dot{\psi}_{A, 2}(t)=\pi h d_{B, k} / T$; see (9). Furthermore, the received phase derivative at node $A$ during the third phase is simply $\dot{\psi}_{A, 3}(t)=\pi h\left(d_{A, k}+d_{B, k}\right) / T . \quad$ As a result, $\dot{\Psi}_{A, 3}(t)=\dot{\psi}_{A, 3}(t)-\dot{\theta}_{A}(t)=\pi h d_{B, k} / T$. This means the sign of the decision variable $D$ in (20) equals the sign of the data bit $d_{B, k}$. Naturally, in the presence of fading and noise, these phase derivatives are subjected to distortions. However, as long as the channel's average signal-to-noise ratio is at a decent level, the decision rule in (22) will still enable us to recover the data reliably. Further discussion on the optimality of (20) can be found in [15]. 


\subsection{Detection of the 3-DPF and A4-DPF signals}

From the discussion in Sections 2.1 and 2.3, we can see that 3-DPF is a specific case of A4-DPF. For both schemes, the relay broadcasts a signal of the form $x_{R}(t)=\exp \left\{j \theta_{R}(t)\right\}$ in the final phase of cooperation, where $\theta_{R}(t)=\left(w_{A} \hat{\theta}_{A}(t)+w_{B} \hat{\theta}_{B}(t)\right)$ is the phase of the relayed signal, $\hat{\theta}_{A}(t)$ and $\hat{\theta}_{B}(t)$ are the decoded phases at the relay, $\left(w_{A}, w_{B}\right)=(1,1)$ for $3-\mathrm{DPF}$, and $\left(w_{A}, w_{B}\right)$ alternates between $(3,1)$ and $(1,3)$ for A4-DPF. Using $(17)$ as the definition of the received signal at A during the third phase, we first remove A's own phase from $\psi_{A, 3}(t)$ according to

$$
\begin{aligned}
& Y_{A, 3}(t)=a_{A, 3}(t) \mathrm{e}^{j \Psi_{A, 3}(t)}, \\
& \Psi_{A, 3}(t)=\left(\psi_{A, 3}(t)-w_{A} \theta_{A}(t)\right) / w_{B} .
\end{aligned}
$$

and then combine the derivative of $\Psi_{A, 3}(t)$ non coherently with $\dot{\psi}_{A, 2}$, the received phase derivative at $A$ in Phase 2, according to (20) and (21). As in the case of PSPF, the decision rule is given by (22).

One nice feature about DF-based strategies is that the modulation index used at the relay, $h_{R}$, needs not to be identical to $h$, the modulation index used by $\mathrm{A}$ and $\mathrm{B}$. This flexibility is especially important if we want to impose stringent bandwidth requirement on the signal transmitted by the relay. If the relay does use a different modulation index, the effective form of the forwarded phase is $\theta_{R}(t)=\rho\left(w_{A} \hat{\theta}_{A}(t)+w_{B} \hat{\theta}_{B}(t)\right)$, where $\rho=h_{R} / h$ is the ratio of modulation indices. In this case, (23) should be modified to

$$
\begin{aligned}
& Y_{A, 3}(t)=a_{A, 3}(t) \mathrm{e}^{j \Psi_{A, 3}(t),} \\
& \Psi_{A, 3}(t)=\left(\psi_{A, 3}(t)-\rho w_{A} \theta_{A}(t)\right) /\left(\rho w_{B}\right) .
\end{aligned}
$$

before combining with $\dot{\psi}_{A, 2}$ according to (20) and (21).

\section{Implementation issues}

We provide in this section some implementation guidelines for the proposed PSPF strategy. Comparison with the considered decode-and-forward schemes, in terms of implementation complexity, will also be made.

According to (10), a PSPF relay needs to first convert the signals $y_{R, 1}(t)$ and $y_{R, 2}(t)$ in (1) and (3) into the constant envelope signals $\hat{\gamma}_{R, 1}(t)=\exp \left\{j \arg \left[\gamma_{R, 1}(t)\right]\right\}$ and $\hat{\gamma}_{R, 2}(t)=\exp \left\{j \arg \left[\gamma_{R, 2}(t)\right]\right\}$ before transmitting the product signal $x_{R}(t)=\hat{y}_{R, 1}(t) \hat{y}_{R, 2}(t)$ in the final phase of relaying. Given that the relay is half-duplex and cannot transmit and receive at the same time, it must first detect and store (the sufficient statistics of) the data packets it receives from $A$ and $B$ in their entireties before generating and forwarding the product constant envelope signal in the final phase. The procedure requires frame synchronization at the relay to ensure proper time-alignment of $\hat{\gamma}_{R, 1}(t)$ and $\hat{\gamma}_{R, 2}(t)$. This can be done by inserting a special sync pattern into each data packet and correlating the received signals with this pattern at the relay. As for storage of the entire frames of $\hat{y}_{R, 1}(t)$ and $\hat{y}_{R, 2}(t)$, this will be done in the digital domain via sampling and quantization. The minimum sampling frequency will be twice the bandwidth of $x_{R}(t)$, rather than twice the bandwidth of individual $\hat{\gamma}_{R, 1}(t)$ and $\hat{y}_{R, 2}(t)$. This stems from the fact that signal mixing (multiplication) is a bandwidth-expanding process. We found that when the two source signals in (1) and (3) (namely $x_{A}(t)$ and $x_{B}(t)$ ) are MSK, then the product signal $x_{R}(t)$ has a bandwidth of $1.832 / T$, where $1 / T$ is the bit rate. Therefore, in this case, a sampling frequency of $4 / T$ would be sufficient to create signal samples that capture all the information about the product signal. As for quantization, it is relatively straight forward because, unlike the original received signals $y_{R, 1}(t)$ and $y_{R, 2}(t)$, the real and imaginary components of $\hat{\gamma}_{R, 1}(t)$ and $\hat{\gamma}_{R, 2}(t)$ all have finite dynamic range. Specifically, the values of these components are confined to the interval $[-1,+1]$. Given the limited dynamic range, we can use a simple $b+1$ bits uniform quantizer, where $b$ is chosen such that the signal-to-quantization noise ratio (SQNR) is much higher than the channel signal-tonoise ratio seen at the destination receiver. Since the SQNR of a uniform quantizer (assuming that the real and imaginary components of $\hat{\gamma}_{R, 1}(t)$ and $\hat{\gamma}_{R, 2}(t)$ are uniformly distributed in $[-1,+1]$ ) varies according to $2^{2}$ $(B+1)$ [18], an 8-bit $(b=7)$ quantizer can already yield a SQNR of $48 \mathrm{~dB}$, which is much higher than the anticipated channel Signal-to-Noise-Ratio (SNR).

From the above discussion, it becomes clear that the proposed PSPF scheme requires a total of

$$
N_{\mathrm{PSPF}}=4 \cdot(b+1) \cdot K \cdot N
$$

bits to store the signals $\hat{y}_{R, 1}(t)$ and $\hat{y}_{R, 2}(t)$ at the relay, where $f_{\mathrm{s}}=\mathrm{K} / \mathrm{T}$ is the sampling frequency, $b+1$ is the number of bits used in quantization, $N$ is the number of bits in each data packet, and the factor of 4 is the total number of real and imaginary components in $\hat{\gamma}_{R, 1}(t)$ and $\hat{\gamma}_{R, 2}(t)$. In contrast, the 3-DPF and A4-DPF schemes described in Sections 2.2 and 2.3 require only

$$
N_{\text {DPF }}=2 N
$$

bits to store the decoded bit streams $\left\{\hat{d}_{A, n}\right\}_{n=1}^{N}$ and $\left\{\hat{d}_{B, n}\right\}_{n=1}^{N}$. However, this reduction in storage 
requirement comes at the expense of additional computations required for demodulation and re-modulation at the relay. According to (21), the discriminator detector used for demodulation needs to compute the phase derivatives in the original received signal $y_{R, 1}(t)$ and $y_{R, 2}$ $(t)$ at the decision making instants. These derivatives can be expressed in terms of the constant envelope signals $\hat{\gamma}_{R, 1}(t)$ and $\hat{\gamma}_{R, 2}(t)$ as $-j \cdot \hat{\gamma}_{R, 1}^{*}(t) \dot{\hat{\gamma}}_{R, 1}(t)$ and $-j \cdot \hat{\gamma}_{R, 2}^{*}(t) \dot{\hat{\gamma}}_{R, 2}(t)$, where $\hat{\gamma}^{*}$ and $\dot{\hat{\gamma}}$ represent respectively signal conjugation and derivative. Let us assume the two signal derivatives $\dot{\hat{\gamma}}_{R, 1}(t)$ and $\dot{\hat{\gamma}}_{R, 2}(t)$ are computed in the digital domain with $\hat{\gamma}_{R, 1}(t)$ and $\hat{\gamma}_{R, 2}(t)$ represented by samples spaced $T / K$ seconds apart, where $K$ is an integer that is large enough to ensure that the sampling frequency $f_{\mathrm{s}}=K / T$ is higher than twice the bandwidth of the product signal $x_{R}(t)=\hat{y}_{R, 1}(t) \hat{y}_{R, 2}(t)$. Then the corresponding discrete-time differentiator is simply a $K$-tap digital finite impulse response filter ${ }^{\mathrm{a}}$ with a computational complexity of $K$ complex multiply-and-add (CMAD) for each decoded bit $\hat{d}_{A, n}$ or $\hat{d}_{B, n}$. As a result, the total demodulation complexity is

$$
C_{\text {DEMOD }}=2 K N(\mathrm{CMAD})
$$

As for the re-modulation complexity in DPF, if we assume a table look-up based modulator, then the basic operations are waveform fetching and concatenation. These operations can be assumed insignificant when compared to the multiply-and-add operations mentioned above. Although a table-look-up re-modulator requires storage of all possible modulation waveforms, this should not be counted towards the storage requirement of the two DPF schemes, since the modulator is always required to transmit a node's own data, irrespective of whether it uses PSPF or DPF while in the relay mode. Another implementation structure that is common to PSPF and DPF is the analog-to-digital converter front end.

In summary, from the computational complexity point of view, PSPF is simpler because it avoids the CMAD operations required for demodulation at the relay. Although it requires substantially more storage, the tradeoff still favors PSPF because memory is inexpensive while additional computational load can, in general, lead to quicker battery drain and even the need of a more powerful processor. We note further that the complexity of PSPF can be further reduced if we adopt direct bandpass processing. This is achieved by first passing $\tilde{y}_{R, 1}(t)$ and $\tilde{y}_{R, 2}(t)$, the bandpass versions of $y_{R, 1}(t)$ and $y_{R, 2}(t)$, through a bandpass filter, followed by bandpass limiting [19], then bandpass sampling [20] and quantization. As shown in [20], the sampling frequency of the bandpass signals is roughly the same as that of their complex baseband versions. Therefore, no high-speed analog to digital converter (ADC) is required. By direct bandpass processing, we can bypass up and down conversions in PSPF altogether, which in turn reduces the number of multiplication and addition required to perform these steps in a digital modulator/demodulator. It should be emphasized that with decode-and-phase-forward, down and up conversion are unavoidable.

\section{Performance analysis}

\subsection{The BER of PSPF}

The BER performance of the proposed PSPF scheme with discriminator detection is evaluated using the characteristic function (CF) approach; see [15]. In the analysis, the variances of the fading processes $g_{A R}(t), g_{B R}(t), g_{A B}(t), g_{B A}(t)$, $g_{R A}(t)$, and $g_{R B}(t)$ in (1) to (6) are denoted as $\sigma_{g_{A R^{\prime}}}^{2} \sigma_{g_{B R}}^{2}, \sigma_{g_{A B}}^{2}, \sigma_{g_{B A}}^{2}, \sigma_{g_{R A}}^{2}$, and $\sigma_{g_{R B}}^{2}$, respectively, with $\sigma_{g_{A R}}^{2}=\sigma_{g_{R A}}^{2} \sigma_{g_{B R}}^{2}=\sigma_{g_{R B}}^{2}$ and $\sigma_{g_{A R}}^{2}=\sigma_{g_{B A}}^{2}$. On the other hand, the variances of the noise processes $n_{R, 1}(t), n_{B, 1}(t), n_{R, 2}(t)$, $n_{A, 2}(t), n_{A, 3}(t)$, and $n_{B, 3}(t)$ in these equations are $\sigma_{n_{R, 1}}^{2}, \sigma_{n_{B, 1}}^{2}, \sigma_{n_{R, 2}}^{2}, \sigma_{n_{B, 2}}^{2}, \sigma_{n_{A, 3}}^{2}$, and $\sigma_{n_{B, 3}}^{2}$, respectively, with $\sigma_{n_{R, 1}}^{2}=\sigma_{n_{B, 1}}^{2}=\sigma_{n_{R, 2}}^{2}=\sigma_{n_{B, 2}}^{2}=N_{0} B_{12}$ and $\sigma_{n_{A, 3}}^{2}=\sigma_{n_{B, 3}}^{2}=N_{0} B_{3}$, where $N_{0}$ is the noise power spectral density (PSD), $B_{12}$ the bandwidth of the receive LPFs in Phases I and II, and $B_{3}$ the bandwidth of the receive LPF in Phase III. In this investigation, $B_{12}$ is always set to the $99 \%$ bandwidth of $x_{A}(t)$ and $x_{B}(t)$, while $B_{3}$ is either the same as $B_{12}$, or set to the $99 \%$ bandwidth of the relay signal $x_{R}(t)$. Given the nature of the symbol-by-symbol detectors described in the previous section, we take the liberty to drop the symbol index $\mathrm{k}$ in $d_{A, k}$ and $d_{B, k}$ in the performance analysis.

First, it is observed that the terms $D_{2}$ in (21) is a quadratic forms of complex Gaussian variables $\left(y_{A, 2}, \dot{y}_{A, 2}\right)$ when conditioned on $\dot{\theta}_{B}$; refer to the Appendix for the statistical relationships between different parameters in the general channel model

$$
\gamma(t)=g(t) \mathrm{e}^{j \theta(t)}+n(t)=a(t) \mathrm{e}^{j \psi(t)},
$$

where $g(t)$ and $n(t)$ are, respectively, $C N\left(0, \sigma_{g}^{2}\right)$ and $C N\left(0, \sigma_{n}^{2}\right), \theta(t)$ is the signal phase, and $a(t)$ and $\psi(t)$ are respectively the amplitude and phase of $y(t)$. Without loss of generality, we assume $d_{B, k}=+1$ and hence $\dot{\theta}_{B}(t)=\pi h / T$. By substituting $\theta=\dot{\theta}_{B}$ into (A5) and (A8), and with $\mathbf{F}$ in (A10) set to the $\left[\begin{array}{ll}0 & -j \\ j & 0\end{array}\right]$ matrix in (21), we can find the two poles of the CF of $D_{2}$ as following:

$$
p_{1}=-\frac{1}{2 \alpha_{A, 2} \beta_{A, 2}\left(1+\rho_{A, 2}\right)}<0, \quad p_{2}=+\frac{1}{2 \alpha_{A, 2} \beta_{A, 2}\left(1-\rho_{A, 2}\right)}>0,
$$


where $\alpha_{A, 2}, \beta_{A, 2}, \rho_{A, 2}$ are determined from (A10) under the conditions $\dot{\theta}=\pi h / T, \sigma_{g}^{2}=\sigma_{g B A}^{2}$, and $\sigma_{n}^{2}=N_{0} B_{12} ; B_{12}$ the bandwidth of the receive filter in Phases I and II.

How about the term $D_{3}$ in (21)? This term can be rewritten as $D_{3}=2 a_{A, 3}^{2} \dot{\Psi}_{A, 3}=2\left(a_{A, 3}^{2} \dot{\psi}_{A, 3}-a_{A, 3}^{2} \dot{\theta}_{A}\right)$, or as

$$
D_{3}=\left(\gamma_{A, 3}^{*} \dot{\gamma}_{A, 3}^{*}\right)\left(\begin{array}{cc}
-2 \dot{\theta}_{A}-j \\
j & 0
\end{array}\right)\left(\begin{array}{l}
y_{A, 3} \\
\dot{y}_{A, 3}
\end{array}\right) \text {, }
$$

which is once again a quadratic form of complex Gaussian variables. This quadratic form, however, depends on a number of parameters. First is the data phase derivation $\dot{\theta}_{A}$. Second, it depends on the forwarded phase derivative $\dot{\theta}_{R}=\dot{\psi}_{R, 1}+\dot{\psi}_{R, 2}$, which in turns depends on both $\dot{\psi}_{R, 1}$ and $\dot{\psi}_{R, 2}$; refer to (16). Of course, $\dot{\psi}_{R, 1}$ depends on $\dot{\theta}_{A}$, while $\dot{\psi}_{R, 2}$ depends on $\dot{\theta}_{B}$, refer to (14) and (15). Note that $D_{2}$ and $D_{3}$ are statistically independent. Conditioned on $\dot{\psi}_{R, 1}, \dot{\psi}_{R, 2}, \dot{\theta}_{A}, \dot{\theta}_{B}=\pi h / T$, and $\mathbf{F}=\left(\begin{array}{cc}-2 \dot{\theta}_{A}-j \\ j & 0\end{array}\right)$, we can determine from (A10) the poles of the CF of $D_{3}$ as

$$
\begin{aligned}
& Q_{1}=\frac{\left(\chi_{A, 3}^{2}-\dot{\theta}_{A} \alpha_{A, 3}^{2}\right)-\sqrt{\alpha_{A, 3}^{2}\left(\dot{\theta}_{A}^{2} \alpha_{A, 3}^{2}-, 2 \dot{\theta}_{A} \chi_{A, 3}^{2}+\beta_{A, 3}^{2}\right)}}{2\left(1-\rho_{A, 3}^{2}\right) \alpha_{A, 3}^{2} \beta_{A, 3}^{2}}<0, \\
& Q_{2}=\frac{\left(\chi_{A, 3}^{2}-\dot{\theta}_{A} \alpha_{A, 3}^{2}\right)+\sqrt{\alpha_{A, 3}^{2}\left(\dot{\theta}_{A}^{2} \alpha_{A, 3}^{2}-2 \dot{\theta}_{A} \chi_{A, 3}^{2}+\beta_{A, 3}^{2}\right)}}{2\left(1-\rho_{A, 3}^{2}\right) \alpha_{A, 3}^{2} \beta_{A, 3}^{2}}>0 .
\end{aligned}
$$

where $\alpha_{A, 3}, \beta_{A, 3}, p_{A, 3}, \chi_{A, 3}^{2}$ are determined from (A10) under the conditions $\dot{\theta}=\dot{\psi}_{R, 1}+\dot{\psi}_{R, 2}, \sigma_{g}^{2}=\sigma_{g_{R A}}^{2}$, and $\sigma_{n}^{2}=N_{0} B_{3} ; B_{3}$ the bandwidth of the receive filter in Phase III.

Recall that we assume $d_{B}=+1$ and hence $\dot{\theta}_{B}(t)=\pi h / T$. In this case, the detector makes a wrong decision when $D<0$. Since the characteristic function of $D$ is $\phi_{D}(s)=\left(p_{1} p_{2}\right)\left(Q_{1} Q_{2}\right) /\left\{\left(s-p_{1}\right)\left(s-p_{2}\right)\left(s-Q_{1}\right)\left(s-Q_{2}\right)\right\}$,

the probability that $D<0$ is the sum of residues of $-\varphi_{D}(s) / s$ at the right plane poles $p_{2}$ and $Q_{2}$, yielding

$$
\operatorname{Pr}\left[D<0 \mid \dot{\theta}_{A}, \dot{\theta}_{B}=\pi h / T, \dot{\psi}_{R_{1},}, \dot{\psi}_{R, 2}\right]=\frac{-p_{1}}{p_{2}-p_{1}} \cdot \frac{Q_{1} Q_{2}}{\left(p_{2}-Q_{1}\right)\left(p_{2}-Q_{2}\right)}+\frac{-Q_{1}}{Q_{2}-Q_{1}} \cdot \frac{p_{1} p_{2}}{\left(Q_{2}-p_{1}\right)\left(Q_{2}-p_{2}\right)} .
$$

Finally, since $\dot{\psi}_{R, 1}$ and $\dot{\psi}_{R, 2}$ are random variables given $\dot{\theta}_{A}$ and $\dot{\theta}_{B}$, respectively, the unconditional error probability can be expressed in semi-analytical form as

$$
\begin{array}{r}
P_{b}=\frac{1}{2} \sum_{d_{A}=-1_{\psi_{R}, 1-\infty}+\infty}^{+1} \int_{\dot{\psi}_{R, 2-\infty}-\infty}^{\infty} \operatorname{Pr}\left[D<0 \mid \dot{\theta}_{A}=\pi h d_{A} / T, \dot{\theta}_{B}=\pi h / T, \dot{\psi}_{R_{2} 1}, \dot{\psi}_{R_{2} 2}\right] \\
p\left(\dot{\psi}_{R, 1} \mid \dot{\theta}_{A}=\pi h d_{A} / T\right) p\left(\dot{\psi}_{R, 2} \mid \dot{\theta}_{B}=\pi h / T\right) d \dot{\psi}_{R, 1} d \dot{\psi}_{R, 2}
\end{array}
$$

where the marginal probability density functions (PDF) $p\left(\dot{\psi}_{R, 1} \mid \dot{\theta}_{A}=\pi h d_{A} / T\right)$ and $p\left(\dot{\psi}_{R, 2} \mid \dot{\theta}_{B}=\pi h / T\right)$ can be determined from (A5) to (A6) in the Appendix.

\subsection{BER of 3-DPF and A4-DPF Signals}

The two multi-level DPF signals broadcasted by the relay in (11) and (12) are constructed from decisions made by the relay about Users $A$ and $B$ 's data. Although different from (10), the exact BER analysis of these signals can still be determined via the characteristic function approach. This stems from the fact that the decision variable $D$ of these DPF schemes are again quadratic forms of complex Gaussian variables when conditioned on the data phase derivatives $\dot{\theta}_{A}$ and $\dot{\theta}_{B}$, as well as their decoded versions $\dot{\hat{\theta}}_{A}$ and $\dot{\hat{\theta}}_{B}$ at the relay. Specifically, the poles of the CF of $D_{2}$ are identical to those in the PSPF case, and can be found in (28). As for the poles of the CF of $D_{3}$, we should first replace the term $\dot{\theta}$ in the Appendix by $\dot{\theta}_{R}=w_{A} \dot{\hat{\theta}}_{A}+w_{B} \dot{\hat{\theta}}_{B}$ and then modify the $\mathbf{F}$ matrix in (A10) to

$$
\mathbf{F}=\left(\begin{array}{cc}
-2 \frac{w_{A}}{w_{B}} \dot{\theta}_{A} & \frac{-j}{w_{B}} \\
\frac{j}{w_{B}} & 0
\end{array}\right) .
$$

The resultant poles are found to be

$$
\begin{aligned}
& Z_{1}=\frac{\left(\chi_{A, 3}^{2}-w_{A} \dot{\theta}_{A} \alpha_{A, 3}^{2}\right)-\sqrt{\alpha_{A, 3}^{2}\left(\left(w_{A} \dot{\theta}_{A}\right)^{2} \alpha_{A, 3}^{2}-2 w_{A} \dot{\theta}_{A} \chi_{A, 3}^{2}+\beta_{A, 3}^{2}\right)}}{2\left(1-\rho_{A, 3}^{2}\right) \alpha_{A, 3}^{2} \beta_{A, 3}^{2}} \cdot w_{B}<0, \\
& Z_{2}=\frac{\left(\chi_{A, 3}^{2}-w_{A} \dot{\theta}_{A} \alpha_{A, 3}^{2}\right)+\sqrt{\alpha_{A, 3}^{2}\left(\left(w_{A} \dot{\theta}_{A}\right)^{2} \alpha_{A, 3}^{2}-2 w_{A} \dot{\theta}_{A} \chi_{A, 3}^{2}+\beta_{A, 3}^{2}\right)}}{2\left(1-\rho_{A, 3}^{2}\right) \alpha_{A, 3}^{2} \beta_{A, 3}^{2}} \cdot w_{B}>0,
\end{aligned}
$$

Where $\alpha_{A, 3}, \beta_{A, 3}, \rho_{A, 3}, \chi_{A, 3}^{2}$ are determined from (A10) under the conditions $\dot{\theta}=w_{A} \dot{\hat{\theta}}_{A}+w_{B} \dot{\hat{\theta}}_{B}, \sigma_{g}^{2}=\sigma_{\text {gRA }}^{2}$, and $\sigma_{n}^{2}=N_{0} B_{3} ; B_{3}$ the bandwidth of the receive filter in Phase III. As in the case of PSPF, the conditional BER is expressed in the form

$$
\operatorname{Pr}\left[D<0 \mid \dot{\theta}_{A}, \dot{\theta}_{B}=\frac{\pi h}{T}, \dot{\theta}_{A}, \dot{\hat{\theta}}_{B}\right]=\frac{-p_{1}}{p_{2}-p_{1}} \cdot \frac{z_{1} z_{2}}{\left(p_{2}-Z_{1}\right)\left(p_{2}-Z_{2}\right)}+\frac{-Z_{1}}{z_{2}-Z_{1}} \cdot \frac{p_{1} p_{2}}{\left(Z_{2}-p_{1}\right)\left(Z_{2}-p_{2}\right)} .
$$

The only difference between (35) and (31) is that the former is conditioned on the hard decisions $\dot{\hat{\theta}}_{A}$ and $\dot{\hat{\theta}}_{B}$ made at the relay, while the latter is based on the soft decisions $\dot{\psi}_{R, 1}$ and $\dot{\psi}_{R, 1}$. If we let $P_{e, A}$ and $P_{e, B}$ be the probabilities that the relay makes a wrong decision about $A$ and $B$ 's data, respectively, then the unconditional BER is

$$
\begin{aligned}
& P_{b}=\frac{1}{2 N_{w}} \sum_{d_{A}=1}^{+1} \sum_{\left|w_{A}, w_{B}\right|}\left\{\left(1-P_{e, A}\right)\left(1-P_{e, B}\right) \operatorname{Pr}\left[D<0 \mid \dot{\theta}_{A}=\pi h d_{A} / T, \dot{\theta}_{B}=\pi h / T, \dot{\hat{\theta}}_{A}=\dot{\theta}_{A}, \dot{\hat{\theta}}_{B}=\dot{\theta}_{B}\right]\right. \\
& +\left(1-P_{e, A}\right) P_{e, B} \operatorname{Pr}\left[D<0 \mid \dot{\theta}_{A}=\pi h d_{A} / T, \dot{\theta}_{B}=\pi h / T, \dot{\hat{\theta}}_{A}=\dot{\theta}_{A}, \dot{\hat{\theta}}_{B}=-\dot{\theta}_{B}\right] \\
& +P_{e, A}\left(1-P_{e, B}\right) \operatorname{Pr}\left[D<0 \mid \dot{\theta}_{A}=\pi h d_{A} / T, \dot{\theta}_{B}=\pi h / T, \dot{\theta}_{A}=-\dot{\theta}_{A}, \dot{\theta}_{B}=\dot{\theta}_{B}\right] \\
& \left.+P_{e, A} P_{e, B} \operatorname{Pr}\left[D<0 \mid \dot{\theta}_{A}=\pi h d_{A} / T, \dot{\theta}_{B}=\pi h / T, \dot{\hat{\theta}}_{A}=-\dot{\theta}_{A}, \dot{\hat{\theta}}_{B}=-\dot{\theta}_{B}\right]\right\}
\end{aligned}
$$

where $N_{w}=1$ for 3-DPF and $N_{w}=2$ for A4-DPF, and the inner summation is over the two different permutations of 
$w_{A}$ and $w_{B}$ in (13). It should be pointed out the error probabilities $P_{e, A}$ and $P_{e, B}$ can be determined by integrating the marginal pdf in (A6) from $-\infty$ to 0 when the data bit is $\mathrm{a}+1$, or from 0 to $+\infty$ when the data bit is a -1 . The end result is of the form $[15,21]$

$$
P_{e, i}=\frac{1}{2}\left(1-\left|\rho_{i}\right|\right) ; \quad i=A, B,
$$

where $\left|\rho_{A}\right|$ and $\left|\rho_{B}\right|$ are $|\rho|$ in (A5) obtained under the conditions $\sigma_{g}^{2}=\sigma_{g_{A R}}^{2}, \sigma_{n}^{2}=N_{0} B_{12} \quad$ and $\sigma_{g}^{2}=\sigma_{g_{B R}}^{2}, \sigma_{n}^{2}=N_{0} B_{12}$, respectively.

\section{Results}

We present next some numerical results for the proposed 2-way 3-phase PSPF and DPF relaying schemes. For simplicity, we only consider the case of minimum shift keying (MSK), i.e., $h=1 / 2$, and plot the BER of the resultant cooperative communication system as a function of the SNR in the direct link between $A$ and $B$. In general, the SNR of a link is defined as the fading variance $\sigma_{g}^{2}$ to noise variance $\sigma_{n}^{2}$ ratio in that link. Since the energy per transmitted bit is $E_{b}=\sigma_{g_{A B}}^{2} T$ and the noise variance is $\sigma_{n}^{2}=N_{0} B_{12}=N_{0} \times 1.1818 / T$ in the direct link, where $N_{0}$ is the noise power spectral density and $1.1818 / T$ is the $99 \%$ bandwidth of MSK, the SNR is equivalent to $0.85 E_{b} / N_{0}$. Unless otherwise stated, all the links are assumed to have the same SNR and the same fade rate $f_{d}$.

Figure 2 considers the case of static fading. Figure 3 considers the case of time-selective fading with a normalized Doppler frequency of $f_{d} T=0.03$ in all the links. To put the 2-way relaying results into perspective, we compare them against the 1-way relaying results from [15] for MSK source signal and phase-forward relay signal. The BER curves shown in these figures were obtained from the semi-analytical expression in (22) and as well as from simulation. The two sets of results are in good agreement.

In the static fading case, it is observed from Figure 2 that 2-way relaying is consistently $3 \mathrm{~dB}$ less power efficient than 1-way relaying over a wide range of BER. In the 'fast' fading case, 2-way relaying has an irreducible error floor around $10^{-3}$ while that of 1 -way relaying sits at $6 \times 10^{-4}$. Above the irreducible error floors and at a BER of $10^{-2}$, the difference between 1 and 2-way relaying is about $5 \mathrm{~dB}$.

One source for the degraded performance stated above is simply energy normalization. In both figures, we assume all the nodes transmit with a bit-energy of $E_{b}$. This means 1 -way relaying needs a total of $4 E_{b}$ to transmit two bits while 2-way relaying needs only $3 E_{b}$ to transmit the same amount of information. Therefore, if we normalize the energy, the difference between the two schemes in the static fading case actually reduces to only $1.5 \mathrm{~dB}$. We regard this loss as acceptable, given that 2-way relaying improves the transmission efficiency by $33 \%$.

The results obtained above were based on using a receive low pass filter (LPF) in the $R-A$ path whose bandwidth, $B_{3}$, equals the $99 \%$ bandwidth of the relay signal. As mentioned earlier, because of the spectral convolution effect, the bandwidth of the relay signal is larger than that of the original MSK signal and is found to be $1.832 / T$. A natural question is, how would PSPF perform if the signal in the $R-A$ path is band-limited to that of the MSK signal? Specifically, what is the tradeoff between a reduced noise figure, but an increased signal distortion because of tighter filtering?

Figure 4 shows the effect of using the same LPF in the relay path and the direct path, i.e., $B_{3}=B_{12}=1.1818 / T$. The simulation results show that with a narrower filter in the relay path, the proposed PSPF scheme actually achieves a better performance. We attribute this to the fact that non-coherent detection is not match filtering, and the reduction in noise level through tighter filtering more than compensates for the self interference that it generates.

In a 3-phase 2-way system, the SNRs of different links are not necessarily equal. For instance, if the relay is much closer to one of $A$ and $B$, then we expect the SNR in the $A R$ or $B R$ link to be higher than that in the $A B$ link. We next show in Figures 5 and 6 BER results for different asymmetric channel conditions, for both static fading and time-selective fading with a normalized fade rate of 0.03. As in Figure 4, the bandwidth of the LPF filter in the $R-A$ path is set to that of MSK. Three different scenarios are considered-(1) all the links have the same SNR, (2) the two source-relay paths have higher SNRs, and (3) only one of the source-relay paths is stronger. Also included in Figures 5 and 6 are the BERs of MSK without diversity and with dual-receive diversity. From the figures, we can see that when the SNR in both the $A-R$ and $B-R$ links is $20 \mathrm{~dB}$ stronger than that in the $A-B$ link, the BER curve exhibits a very prominent second order diversity effect. In contrast, when all the three links are equally strong, the diversity effect disappears (the case when only the $A R$ link has a higher SNR than the $A-B$ link falls in between these two cases).

Finally, we show in Figures 7 and 8 BER curves for the decode-and-forward based 3-DPF and A4-DPF schemes. Also included in the figures are results for the proposed PSPF scheme. The bandwidth of all the receive LPFs is set to $1.1818 / T$, the bandwidth of MSK. From Figure 7, we can see that the performance of PSPF is consistently $2 \mathrm{~dB}$ more energy efficient than the two multi-level DPF schemes when fading is static. With time-selective 


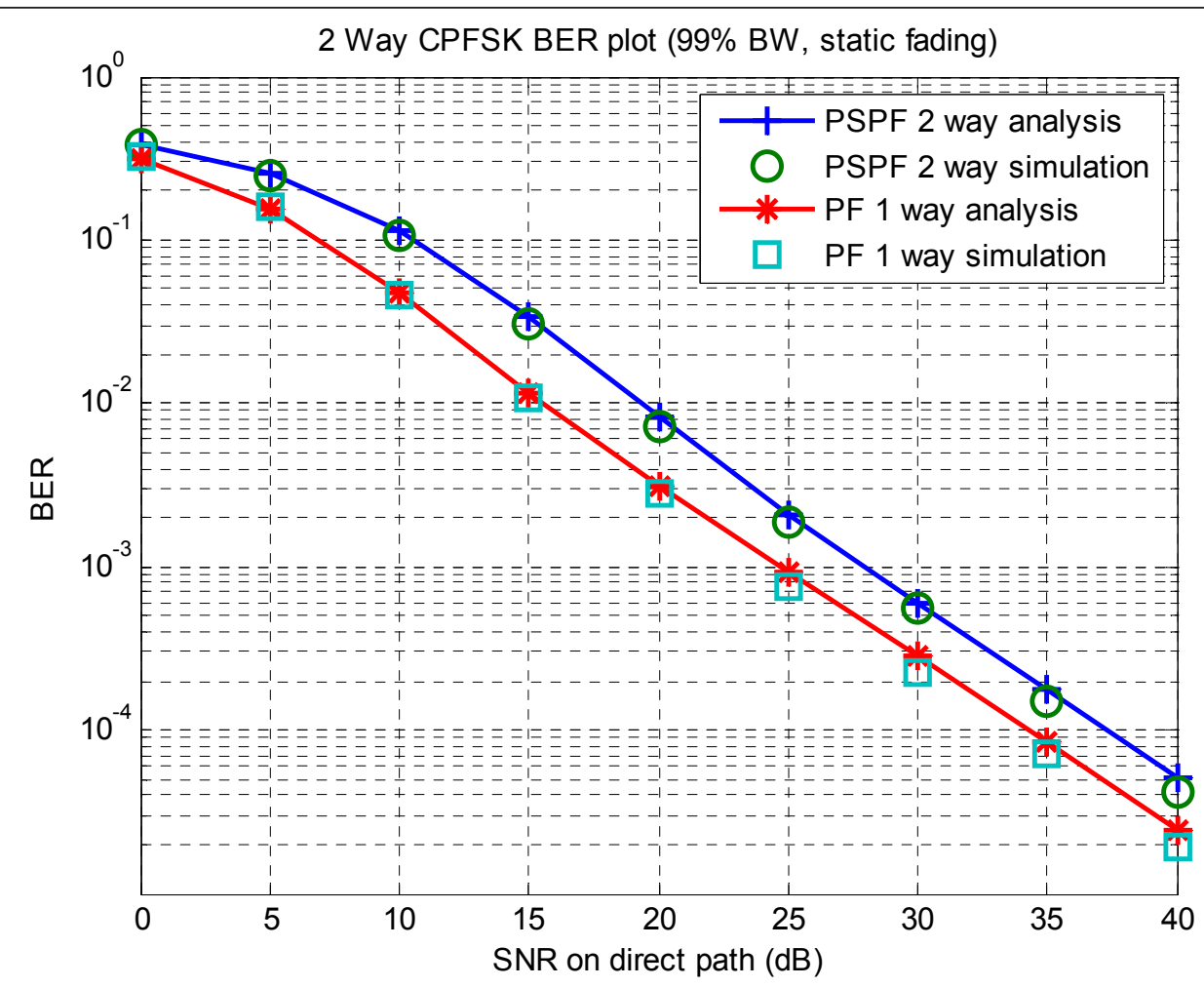

Figure 2 BER of PSPF 2-way 3-phase cooperative transmission in a static Rayleigh fading channel; $B_{12}=1.1818 / T$ and $B_{3}=1.832 / T$.

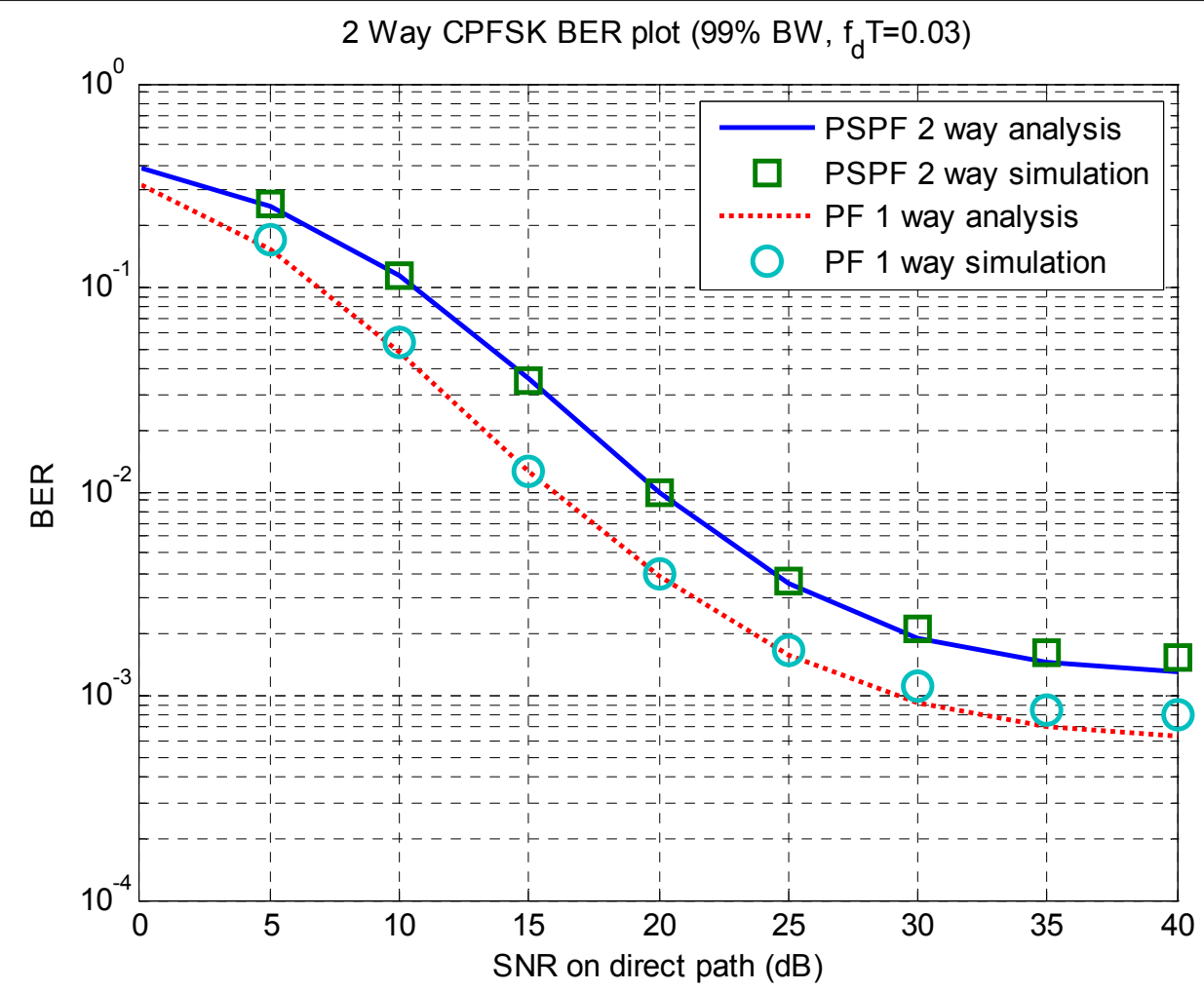

Figure 3 BER of PSPF 2-way 3-phase cooperative transmission in a time-selective Rayleigh fading channel; $f_{d} T=0.03 ; B_{12}=1.1818 / T$ and $B_{3}=1.832 / T$. 


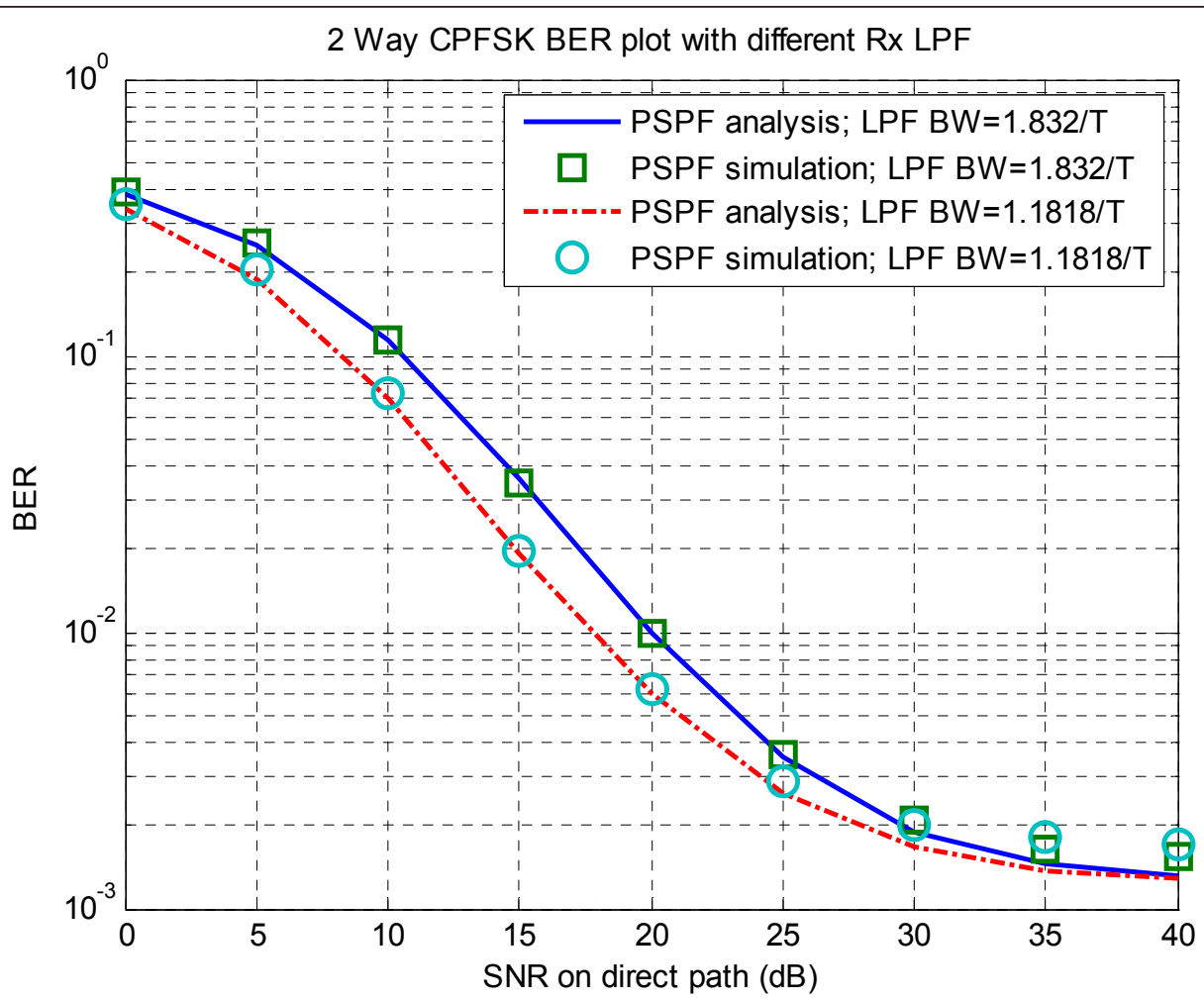

Figure 4 Effect of using the different LPF bandwidth, $B_{3}$, in Phase 3 of PSPF 2-way 3-phase cooperative transmission; $f_{d} T=0.03$

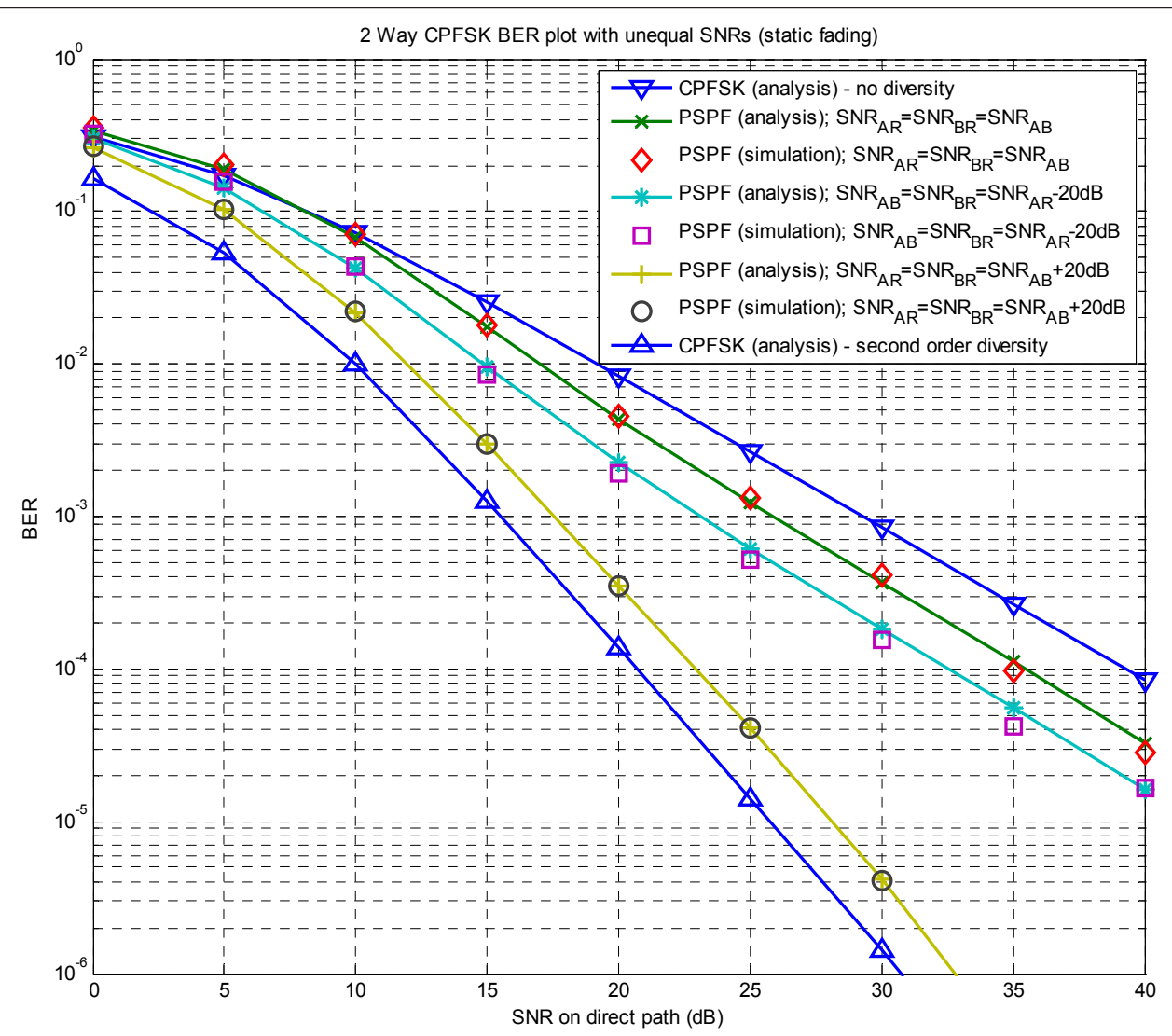

Figure 5 BER at $B$ for unequal SNR under static fading; $\mathrm{SNR}_{A R}$, $\mathrm{SNR}_{B R}$, and $\mathrm{SNR}_{A B}$ are the SNR's in the $A-R, B-R$, and $A-B$ links 


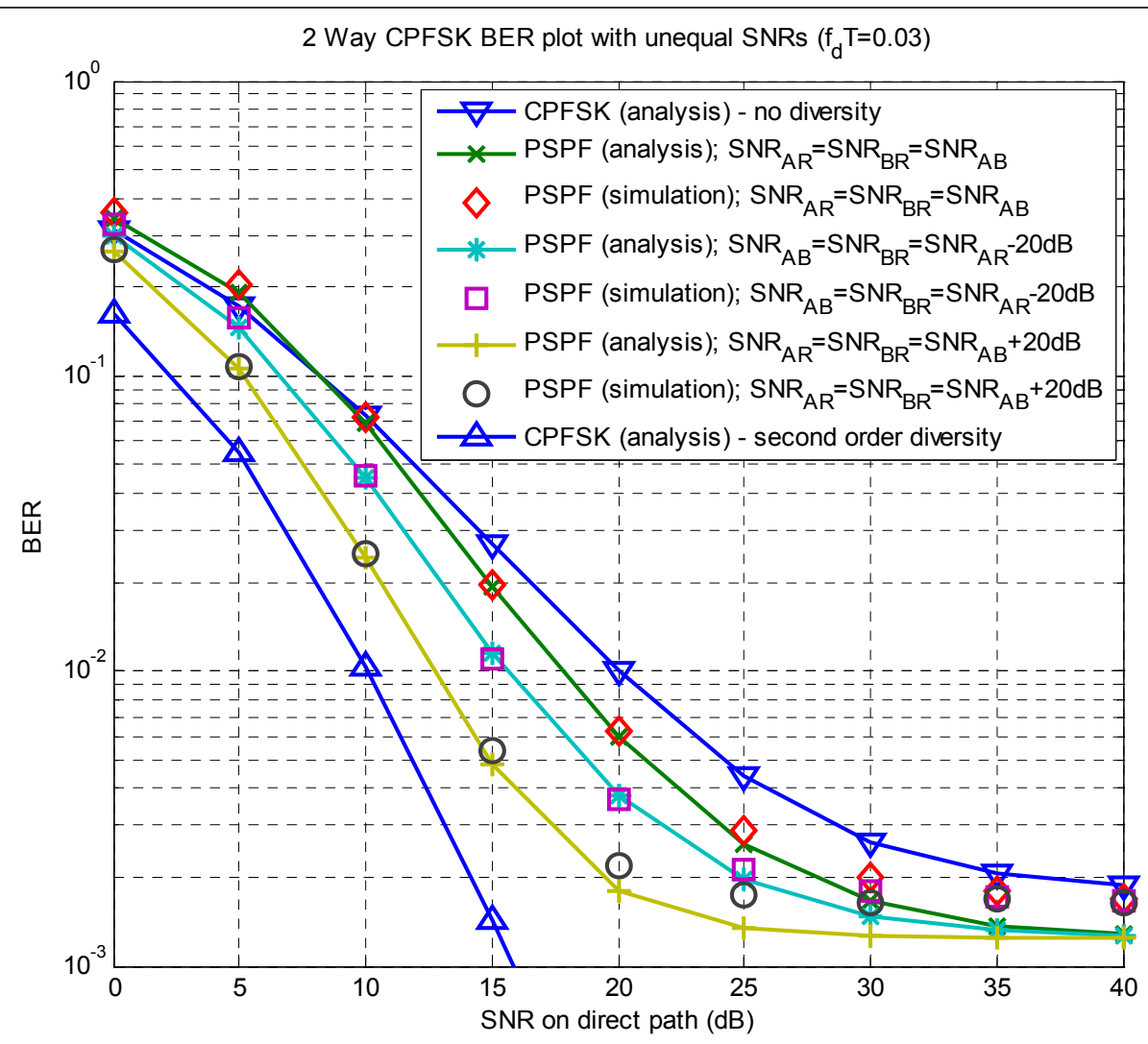

Figure 6 BER at $B$ for unequal SNR and a fade rate of $f_{d} T=0.03$; SNR AR $_{\text {, SNR }}$, and $\mathrm{SNR}_{A B}$ are the SNR's in the $A-R, B-R$, and $A-B$ links.

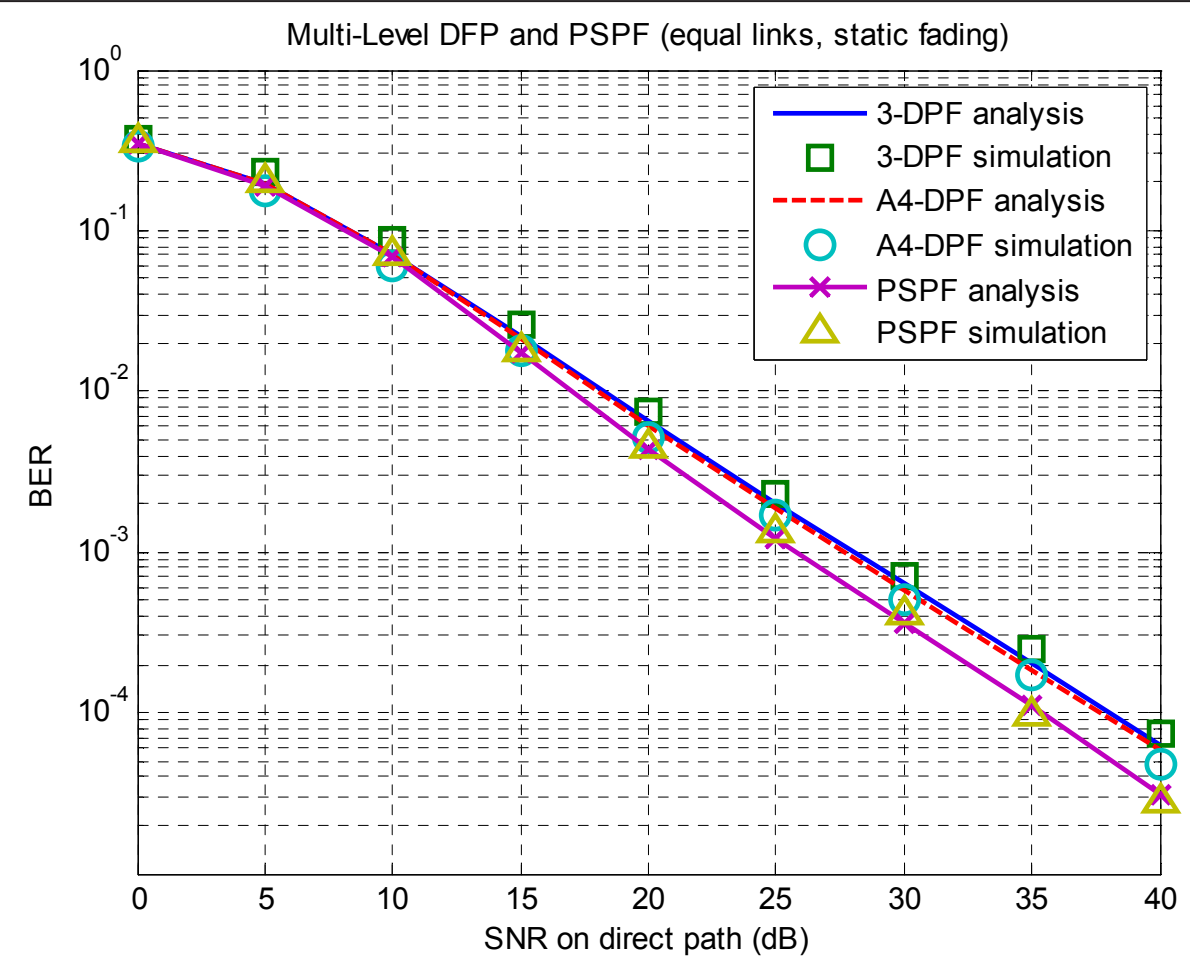

Figure 7 Performance of multi-level DPF and PSPF in static fading channel; $B_{12}=B_{3}=1.1818 / T$. 


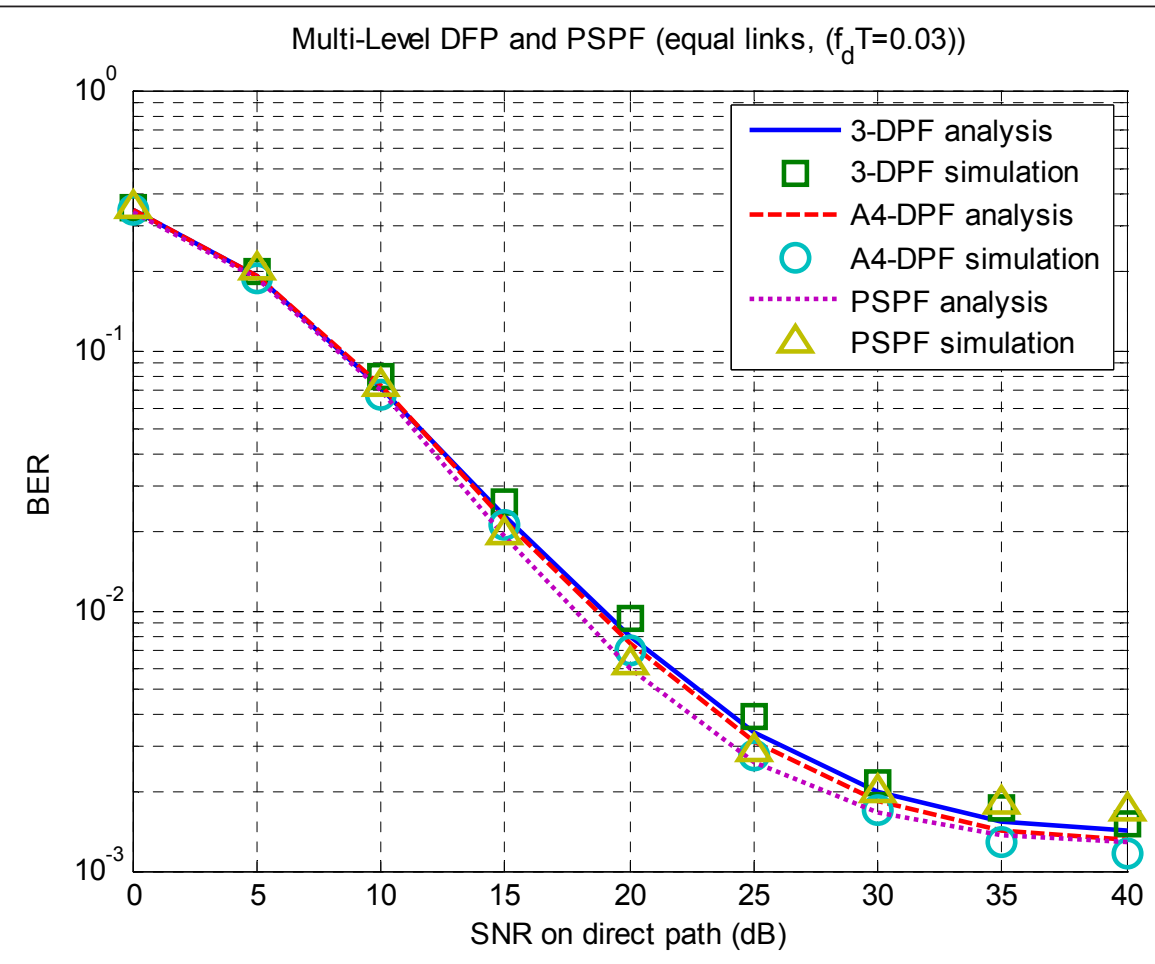

Figure 8 Performance of multi-level DPF and PSPF in a time-selective fading channel with an $f_{d} T=0.03 ; B_{12}=B_{3}=1.1818 / T$.

fading, the simulation results indicate PSPF and A4-DPF have somewhat similar performance and both are more power efficient than 3-DPF. Hence, it can be concluded that the proposed PSPF scheme not only offers a complexity advantage over multi-level DPF, it also provides a BER advantage.

\section{Conclusions}

We consider in this article the use of constant envelope modulation in 2-way 3-phase cooperative transmission. Specifically, a technique referred to as PSPF is proposed and its BER performance compared to 1-way relaying and to 2-way relaying based on decode-and-forward and multi-level re-modulation. As demonstrated in the paper, the proposed technique allows us to maintain constant envelope signaling throughout the signaling chain and does not require complicated signal processing at the relay like its decode-and-forward counterparts. Through analytical and simulation studies, we found that the BER of PSPF with discriminator detection in Rayleigh fading suffers only a moderate loss in energy efficiency (of $1.5 \mathrm{~dB}$ after energy normalization) when compared to its 1-way relaying counterpart. We consider this loss as acceptable, considering that PSPF improves the transmission efficiency by $33 \%$ and it offers a way to avoid expensive linear power amplifiers and complicated signal processing at the relay. We also found that, in comparison with its decoded and forward counterparts, the proposed PSPF scheme offers a lower BER, while at the same time relieves the relay from performing unnecessary demodulation and re-modulation tasks.

\section{Appendix}

We discuss in this Appendix the statistical properties of the faded signal

$$
\gamma(t)=g(t) e^{j \theta(t)}+n(t)=a(t) e^{j \psi(t)},
$$

where $g(t)$ and $n(t)$ are zero-mean complex Gaussian processes with variances (per dimension) of $\sigma_{g}^{2}$ and $\sigma_{n}^{2}$, respectively, $\theta(t)$ the transmitted signal phase, which is treated as a 'deterministic' parameter, and $a(t)$ and $\Psi(t)$, respectively, the amplitude and phase of $y(t)$. Furthermore, the autocorrelation functions of $g(t)$ follows a Jakes spectrum, that is

$$
R_{g}(\tau)=\frac{1}{2} E\left[g^{*}(t) g(t+\tau)\right]=\sigma_{g}^{2} J_{0}\left(2 \pi f_{d} \tau\right)
$$

where $f_{d}$ is the bandwidth (Doppler frequency) of $g(t)$. The noise term, $n(t)$, on the other hand, is band-limited white noise with an autocorrelation function of

$$
R_{n}(\tau)=\frac{1}{2} E\left[n^{*}(t) n(t+\tau)\right]=\sigma_{n}^{2} \operatorname{sinc}(B \tau)
$$

where $\sigma_{n}^{2}=N_{0} B, N_{0}$ being the power spectral density of $n(t)$, and $B$ the bandwidth of $\mathrm{e}^{j \theta(t)}$. 
At any time instant, the joint pdf of $a$, its derivative $\dot{a}, \psi$ its derivative $\dot{\psi}$, given the data phase derivative $\dot{\theta}$, is $[15,22]$

$$
\begin{aligned}
p(a, \dot{a}, \psi, \dot{\psi} \mid \dot{\theta}) & =\frac{a^{2}}{4 \pi^{2} \alpha^{2} \beta^{2}\left(1-\rho^{2}\right)} \exp \left\{-\frac{\dot{a}^{2}}{2 \beta^{2}\left(1-\rho^{2}\right)}\right\} \\
& \times \exp \left\{-\frac{a^{2}}{2 \beta^{2}\left(1-\rho^{2}\right)}\left[\left(\dot{\psi}-\rho \frac{\beta}{\alpha}\right)^{2}+\frac{\beta^{2}}{\alpha^{2}}\left(1-\rho^{2}\right)\right]\right\},
\end{aligned}
$$

Where

$$
\begin{array}{ll}
\alpha^{2}=\frac{1}{2} E\left[|y(t)|^{2}\right]=\sigma_{g}^{2}+\sigma_{n}^{2}, & \beta^{2}=\frac{1}{2} E\left[|\dot{y}(t)|^{2}\right]=\sigma_{g}^{2} \dot{\theta}^{2}+\lambda+\sigma_{\dot{n}}^{2}, \\
\lambda=\frac{1}{2} E\left[|\dot{g}(t)|^{2}\right]=2 \pi^{2} f_{d}^{2} \sigma_{g}^{2}, & \sigma_{\dot{n}}^{2}=\frac{1}{2} E\left[|\dot{n}(t)|^{2}\right]=\pi^{2} B^{2} \sigma_{n}^{2} / 3, \\
\chi^{2}=j \frac{1}{2} E\left[\gamma(t) \dot{\gamma}^{*}(t)\right]=\sigma_{g}^{2} \dot{\theta}, & \rho=\chi^{2} /(\alpha \beta)=\sigma_{g}^{2} \dot{\theta} /(\alpha \beta),
\end{array}
$$

with $\dot{g}(t), \dot{n}(t), \dot{\gamma}(t)$ being the derivatives of $g(t), n(t)$, $y(t)$, respectively. A useful marginal pdf of (A4) is the pdf of $\dot{\psi}$ given $\dot{\theta}$, which is found to be

$$
p(\dot{\psi} \mid \dot{\theta})=\frac{\beta^{2}\left(1-\rho^{2}\right)}{2 \alpha^{2}}\left[\left(\dot{\psi}-\rho \frac{\beta}{\alpha}\right)^{2}+\frac{\beta^{2}}{\alpha^{2}}\left(1-\rho^{2}\right)\right]^{-3 / 2}
$$

Another useful property is that the random vector

$$
\mathbf{r}=\left(\begin{array}{l}
y \\
\dot{y}
\end{array}\right)
$$

is zero mean complex Gaussian with a covariance matrix of

$$
\boldsymbol{\Phi}=\frac{1}{2} E\left[\mathbf{r r}^{\dagger}\right]=\left(\begin{array}{ll}
\alpha^{2} & -j \chi^{2} \\
j \chi^{2} & \beta^{2}
\end{array}\right)
$$

Consequently, the $\mathrm{CF}$ of the quadratic form

$$
D=\mathbf{r}^{\dagger} \mathbf{F r}
$$

is

$$
\phi(s)=\|\mathbf{I}+2 s \boldsymbol{\Phi} \mathbf{F}\|^{-1}=\frac{p_{1} p_{2}}{\left(s-p_{1}\right)\left(s-p_{2}\right)},
$$

where $\|\cdot\|$ denotes the determinant of a matrix, $s$ the transform domain parameter, and $p_{1}<0$ and $p_{2}>0$, respectively, the left and right plane poles of the CF.

\section{Endnotes}

${ }^{a}$ Note that in theory, we can use a higher $(>K)$ order differentiator to improve the accuracy of the derivative estimate. However, it is unclear if this is actually beneficial in practice, given that such a differentiator inevitably involves using samples that span multiple bits and the bit transitions may actually degrade the accuracy of the derivative estimate at the decision making instants.

\section{List of abbreviations}

AF: amplify-and-forward; ADC: analog to digital converter; A4-DPF: alternate 4-level decode-and-phase-forward; AWGN: additive white Gaussian noise; BER: bit-error-rate; CF: characteristic function; CPFSK: continuous-phase frequency-shift-keying; DF: decode-and-forward; DSF: decode-superpositionforward; 3-DPF: 3-level decode-and phase-forward; DXF: decode-XORforward; LPF: low-pass filters; MIMO: multiple-input-multiple-output; MSK: minimum shift keying; PSD: power spectral density; PDF: probability density function; PSPF: phase-superposition-phase-forward; SQNR: signal-toquantization noise ratio.

\section{Acknowledgements}

This work was supported by the Natural Sciences and Engineering Research Council (NSERC) of Canada. It was presented in parts at VTC 2010F in Ottawa, Canada, Sept 2010.

\section{Competing interests}

The authors declare that they have no competing interests.

Received: 14 December 2010 Accepted: 6 October 2011 Published: 6 October 2011

\section{References}

1. A Nosratinia, TE Hunter, A Hedayat, Cooperative communication in wireless network. IEEE Commun Mag. 42(10), 74-80 (2004). doi:10.1109/ MCOM.2004.1341264

2. A Sendonaris, E Erkip, B Aazhang, User cooperative diversity-part l: system description. IEEE Trans Commun. 51(11), 1927-1938 (2003). doi:10.1109/ TCOMM.2003.818096

3. JN Laneman, DNC Tse, GW Wornell, Cooperative diversity in wireless networks: efficient protocols and outage behavior. IEEE Trans Inf Theory 50(12), 3062-3080 (2004). doi:10.1109/TIT.2004.838089

4. A Ribeiro, XD Cai, GB Giannakis, Symbol error probabilities for general cooperative links. IEEE Trans Wireless Commun. 4(3), 1264-1273 (2005)

5. YD Jing, $\mathrm{H}$ Jafarkhani, Single and multiple relay selection schemes and their achievable diversity orders. IEEE Trans Wireless Commun. 8(3), 1414-1423 (2009)

6. Y Zhao, R Adve, TJ Lim, Improving amplify-and-forward relay networks: optimal power allocation versus selection. IEEE Trans Wireless Commun. 6(8), 3114-3123 (2007)

7. RU Nabar, H Bolcskei, FW Kneubuhler, Fading relay channels: performance limits and space-time signal design. IEEE J Select Areas Commun. 22(6), 1099-1109 (2004). doi:10.1109/JSAC.2004.830922

8. SJ Kim, P Mitran, V Tarokh, Performance bounds for bidirectional coded cooperation protocols. IEEE Trans Inf Theory 54(11), 5235-5241 (2008)

9. XJ Zhang, Y Gong, Adaptive power allocation in two-way amplify-andforward relay networks, in IEEE ICC'O9 (August 2009)

10. ZH Yi, IM Kim, Optimum beamforming in the broadcasting phase of bidirectional cooperative communication with multiple decode-and-forward relays. IEEE Trans Wireless Commun. 8(12), 5806-5812 (2009)

11. QF Zhou, YH Li, FCM Lau, B Vucetic, Decode-and-forward two-way relaying with network coding and opportunistic relay selection. IEEE Trans Commun. 58(11), 3070-3076 (2010)

12. $M$ Chen, A Yener, Power allocation for multiple access two-way relaying, in Proceedings of IEEE ICC'O9, Dresden, Germany, (June 2009)

13. RL Maw, PA Martin, DP Taylor, Cooperative relaying with CPFSK and distributed space-time trellis codes. IEEE Commun Lett. 12(5), 356-358 (2008)

14. MC Valenti, D Torrieri, T Ferrett, Noncoherent physical-layer network coding using binary CPFSK modulation, in Millcom 2009 (October 2009)

15. Q Yang, P Ho, Cooperative transmssion with continuous phase frequency shift keying and phase-only forward relays, in Proceedings of IEEE Globecom'09 Hawaii, USA, (December 2009)

16. JB Anderson, T Aulin, C-EW Sundberg, Digital Phase Modulation (Plenum, New York, 1986)

17. JG Proakis, Digital Communications, 4th edn. (McGraw Hill, New York, 2000)

18. AV Oppenheim, RW Schafer, Discrete-Time Signal Processing, 2nd edn. (Prentice Hall, New Jersey, 1998) 
19. J Max, Envelope Fluctuation in the Output of a Bandpass Limiter. IEEE Trans Commun Technol. COM-18(5), 597-605 (1970)

20. RG Vaughan, NL Scott, DR White, The Theory of Bandpass Sampling. IEEE Trans Signal Process. 39(9), 1973-1984 (1991). doi:10.1109/78.134430

21. P Ho, JH Kim, Pilot symbol-assisted detection of CPM schemes operating in fast fading channels. IEEE Trans Commun. 44(3), 337-347 (1996). doi:10.1109/26.486328

22. SM Elnoubi, Analysis of GMSK with discriminator detection in mobile radio channel. IEEE Trans Veh Technol. VT-35(2), 71-76 (1986)

doi:10.1186/1687-1499-2011-120

Cite this article as: Tan and Ho: Two-way relaying using constant envelope modulation and phase-superposition-phase-forward. EURASIP Journal on Wireless Communications and Networking 2011 2011:120.

\section{Submit your manuscript to a SpringerOpen ${ }^{\circ}$ journal and benefit from:}

- Convenient online submission

- Rigorous peer review

- Immediate publication on acceptance

- Open access: articles freely available online

- High visibility within the field

- Retaining the copyright to your article

Submit your next manuscript at $\gg$ springeropen.com 